\title{
Recipient site preparation for epidermal graft in stable vitiligo by a special fraise*
}

\section{Ali Ebrahimi ${ }^{1}$}

\author{
Mohammad Radmanesh ${ }^{2}$
}

Hossein Kavoussi ${ }^{1}$

\section{DOI: $h$ ttp://dx.doi.org/10.1590/abd1806-4841.20153205}

\begin{abstract}
BACKGROUND: The only approach used in the refractory lesions of stable vitiligo is the surgical supply of melanocytes. Suction Blistering Epidermal Graft is one of the most common and effective techniques. There are multiple modalities, including the motor-driven diamond fraise, for the preparation of recipient sites in suction blistering epidermal graft with different repigmentation rates and complications.

OBJECTIVES: To evaluate preparation of recipient site by a motor-driven dental lab finishing carbide bur.

MetHods: Sixty-one stable, depigmented lesions were selected in 14 patients (9 women and 5men), aged 16-29 years, of which 9, 3 and 2 had localized, generalized and segmental vitiligo, respectively. Recipient site was prepared by a motor-driven dental lab finishing carbide bur.

RESULT: Excellent repigmentation at the recipient site was observed in 53 out of 61 (86.9\%) grafted lesions. Postinflammatory hyperpigmentation and perigraft halo were seen in $11(18 \%)$ and $17(27.9 \%)$ patients at the recipient site, respectively.

CONCLUSION: Using a motor-driven dental lab finishing carbide bur to prepare the recipient site of suction blistering epidermal graft technique is reliable and effective, removing only the depigmented epidermis in a simple and safe manner, even on complex-shaped lesions and scar-prone sites.
\end{abstract}

Keywords: Dermabrasion; Graft survival; Vitiligo

\section{INTRODUCTION}

Vitiligo is a relatively common pigmentary disorder but despite multiple medical modalities, partial repigmentation occurs in most patients due to a deficiency in the reservoir of melanocytes. Various surgical modalities include tissue grafts; cultured or noncultured cellular grafts are frequently used to provide a melanocyte reservoir for repigmentation of refractory lesions in patients with stable vitiligo and other conditions involving permanent loss of melanocytes, such as burn leucoderma. ${ }^{1-7}$ Suction Blistering Epidermal Graft (SBEG) is one of the most efficient and successful surgical modalities. ${ }^{6}$ It entails three phases: harvesting of an intact epidermis containing viable melanocytes from a donor site by sustained negative pressure; preparation of a recipient site; and transplantation of epidermal graft to the recipient site. ${ }^{2,6,7}$ There are several modalities for preparing the recipient site in SBEG, including: liquid nitrogen(LN), topical 8-methoxy psoralen followed by ultraviolet A (topical PUVA), suction pumps, motor- driven diamond fraise, $\mathrm{Co}_{2}$ and Erbium-YAG lasers and ultrasonic surgical aspirators. ${ }^{1-8}$

Each modality has advantages and disadvantages. The disadvantages include: partial or complete graft failure, color mismatch, post-inflammatory hyperpigmentation (PIH) in recipient area, scar formation and incomplete removal of recipient epidermis. ${ }^{15,6,8}$

In this study, we used a motor-driven dental lab carbide bur instead of a routine diamond fraise to prepare the recipient site. Interestingly, despite its coarser appearance in comparison with the diamond fraise, this carbide bur only removes the epidermis (Figures 1 and 2). We evaluated the repigmentation rates at different recipient sites as surgical outcomes and complications of this method.

\footnotetext{
Received on 10.10.2013.

Approved by the Advisory Board and accepted for publication on 16.03.2014. IRCT138802251906N1).

Financial Support: None

Conflict of Interest: None

Kermanshah University of Medical Sciences (KUMS) - Kermanshah, Iran.

Ahvaz Jundishapur University of Medical Sciences (AJUMS) - Ahvaz, Iran.

Work performed at the Hajdaiee dermatology clinic, Kermanshah University of Medical Sciences (KUMS). The study protocol conformed to the guidelines of the 1975 Declaration of Helsinki. Our Institutional Review Board and Ethics Committee approved this study. This study was registered in IRCT ( Irct ID: 


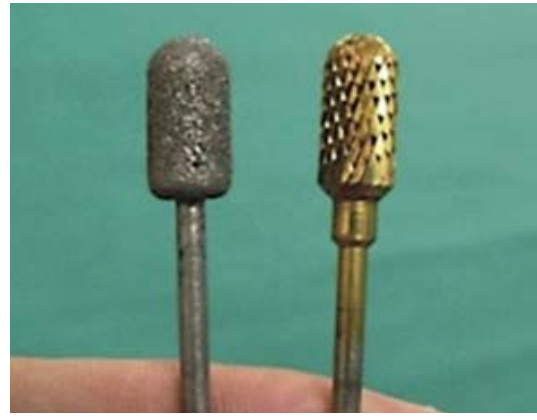

FIGURE 1:

Carbide bur on right side and diamond fraise on left side

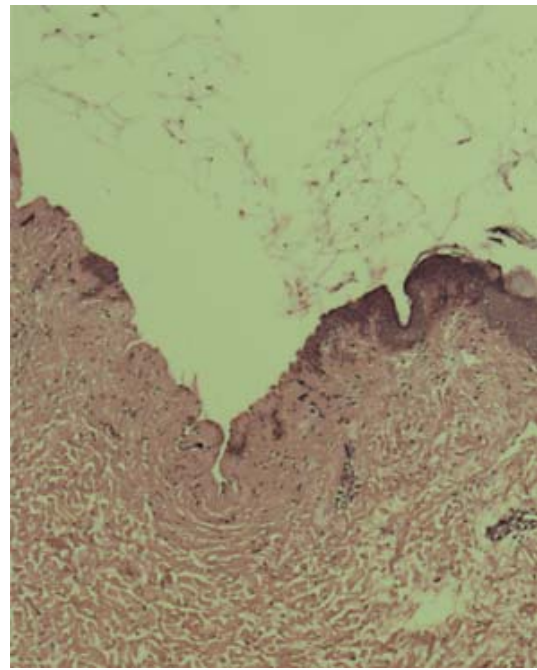

Figure 2:

Chest wall skin tissue immediately after dermabrasian, removed epiderm in central portion and unremarkable dermis (H\&E stain $\mathrm{X} 400)$

\section{PATIENTS AND METHODS}

Sixty-one stable, depigmented lesions were selected in 14 patients ( 9 women and 5 men), with a mean age of 23.2 years (SD 4.29, range 16-33). Nine patients had localized vitiligo, 3 had generalized vitiligo, while 2 had segmental vitiligo, all of which had been unresponsive to medical therapeutic modalities such as PUVA, narrow band UVB and topical steroids for at least 6-8 months (Table 1).

All vitiligo patients included in our study had a segmental and/or stable disease for over a year with negative history of Kobner phenomenon. Exclusion criteria included: unstable vitiligo, positive Kobner phenomenon, pregnancy, aged under 15 years, universal vitiligo, and repair disorders such as kelloid formers.

The characteristics of recipient sites are outlined in table 1. Pre- and postoperative photographs of recipient sites were taken.

The donor site was cleaned with povidone iodine $10 \%$. After using 1-2\% lidocaine for local anesthesia of anterolateral of leg as a donor site, we used three-way connected double syringes (funnel cylinder technique) to harvest epidermal grafts. ${ }^{2}$ Sustained negative pressure, induced by air aspiration via
TABLE 1: Characteristics of clinical type and location of treatment in 14 patients

\begin{tabular}{llll}
\hline Variables & & Frequency & Percentage \\
\hline Clinical & Localized & 9 & 64.3 \\
type $(\mathrm{n}=14)$ & Diffuse & 3 & 21.5 \\
& Segmental & 2 & 14.2 \\
Location of & Hand & 17 & 27.9 \\
treatment $(\mathrm{n}=61)$ & Forehead & 10 & 16.4 \\
& Chest & 5 & 8.2 \\
& Eye lids & 5 & 8.2 \\
& Chin & 4 & 6.5 \\
& Abdomen & 3 & 4.9 \\
& Fore foot & 3 & 4.9 \\
& Finger & 3 & 4.9 \\
& Thigh & 3 & 4.9 \\
& Forearm & 2 & 3.3 \\
& Leg & 2 & 3.3 \\
& Lumbosacral & 2 & 3.3 \\
& region & & \\
& Cheek & 1 & 1.6 \\
& Neck & 1 & 1.6 \\
& Good to & 6 & 9.8 \\
& Moderate & & \\
& Poor & 2 & 3.3 \\
& Post-inflammatory & 11 & 18 \\
& hyperpigmentation & & \\
& Infection & 0 & 0 \\
\hline
\end{tabular}

syringe or funnel, was maintained by locking threeway, leading to development of a blister at the donor site after 1.5-2.5 hours.

The recipient site was cleaned using povidone iodine $10 \%$ and anesthetized with $1-2 \%$ lidocaine without epinephrine. Depigmented epidermis was removed by a motor-driven dermabrader, using a cylindrical, round end, titanium nitride-coated carbide bur with acute angle cutting edges and 36 flutes (CHAMPION SC1 CYLINDER RADIUS END CARBIDE BUR - Titanium Nitride Coated). Forward and backward movements of the hand piece using 10000$15000 \mathrm{rpm}$, with gentle pressure of the carbide bur on the epidermis while holding the hand piece and carbide bur parallel to the skin surface, led to removal of depigmented epidermis (Figures 3 and 4). We ceased further abrasion after the appearance of pinpoint bleedings, a superficial indicator of papillary dermis.

The roof of the blister was removed using Iris scissors; the dermal surface of harvested epidermis was then placed over the abraded recipient site (Figure 5). Several openings were made by needle tip in the larger grafted epidermis at the recipient site to prevent separation of the grafted epidermis, by oozing from the recipient site. The grafts were held in place on the recipient site with non-adherent dressing and fixed with sterilized gauze and adhesive bandage or tape for 7-10 days. All patients were advised to keep the recipient site immobile for at least 7-10 days. We used hand splints for hand and finger immobiliza- 
tion. The dressing was checked for any sign of infection after 4-5 days by removing the adhesive bandage and superficial layers of gauze carefully, while the non-adherent dressing and innermost layers of gauze were left over the grafted epidermis for 7-10 days.

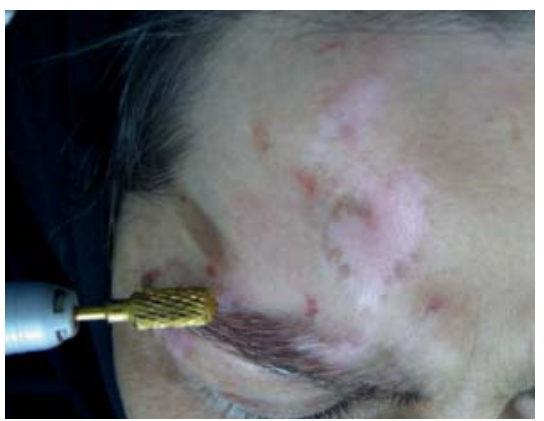

Figure 3:

Patient with vitiligenous lesions in forehead and upper lid

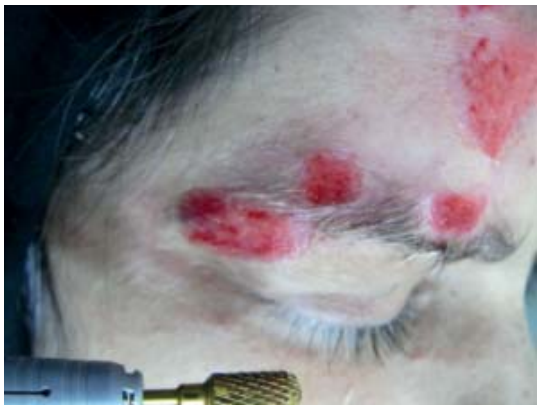

Figure 4:

Recipient site preparation by carbide bur

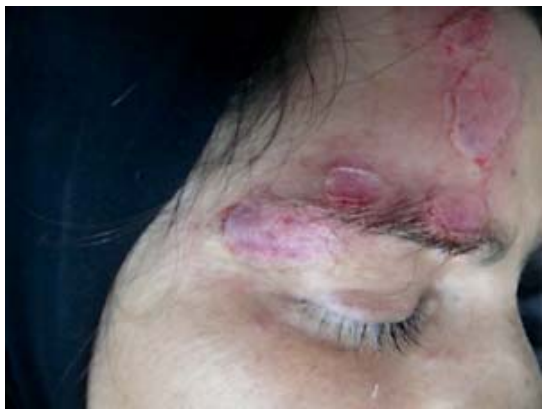

FigURE 5:

Blister roof transplantation, note complete coverage of epidermal graft

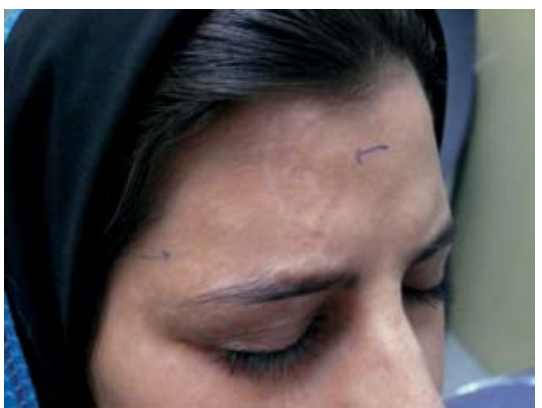

FIGURE 6:

Excelle $\mathrm{nt}$ repigmentation in grafted skin
Several skin biopsies were performed immediately after dermabrasion from the edge of the recipient site.

The repigmentation rate of lesions was classified as excellent (over $75 \%$ repigmentation), good to moderate (25-75\% repigmentation), and poor (under $25 \%$ repigmentation) (Figure 6).

All patients were visited every week in the first month and once a month for the following 6-8 months, for likely complications, such as perigraft halo and PIH at recipient sites, keloid formation, infection and repigmentation rate.

Perigraft halo is defined as the remaining depigmented area in the periphery of grafted skin, due to shrinkage of grafted epidermis or incomplete preparation of recipient site and/or incomplete adherent of grafted epidermis at the periphery.

Written informed consent was obtained from all patients. The study protocol conformed to the guidelines of the 1975 Declaration of Helsinki. Our Institutional Review Board and Ethics Committee approved this study. This study was registered in IRCT (Irct ID: IRCT138802251906N1)

Data analysis was carried out using the SPSS software (version 16) and performed via the Chisquare or Fisher's exact test, to determine the association between treatment outcome and complication by location of lesions and clinical type.

\section{RESULTS}

Of the 14 treated patients, 4 (28.6\%), 7 (50\%) and $3(21.4 \%)$ had types 2,3 and 4 phototype skin, respectively.

Repigmentation began within 3 to 5 weeks of grafting and pigment spreading occurred during the follow-up period. Excellent repigmentation at the recipient site was observed in 53 out of $61(86.9 \%)$ grafted lesions (Figure 5). Good to moderate repigmentation was observed in $6(9.8 \%)$ and poor repigmentation in $2(3.3 \%)$ of the grafted lesions, respectively (Table 2).

The best repigmentation was noted in vitiligeneous lesions in the hands, forehead, eyelids, chin, forefoot, fingers, forearm, cheek and neck, while moderate to good repigmentation was seen in the lumbosacral and chest areas (Table 2).

Both perigraft halo and PIH were seen, respectively, in $17(27.9 \%), 11(18 \%)$ and $4(6.56 \%)$ of the repigmented lesions in the first two months after grafting but they cleared completely after 6-8 months (Tables 3 and 4).

Scar and keloid formations were not observed on recipient sites. No recurrence of the vitiligo at the grafted sites or reactivation of vitiligo was noted within the follow-up period. The Koebner phenomenon and infection were not observed at the donor and/or 
TABLE 2: Outcome of treated lesions based on location

\begin{tabular}{lllcll}
\hline & Excellent & $\begin{array}{c}\text { Good- } \\
\text { Moderator }\end{array}$ & poor Total \\
\hline Location & Forehead & 10 & 0 & 0 & 10 \\
& Eyelids & 5 & 0 & 0 & 5 \\
& Cheek & 1 & 0 & 0 & 1 \\
& Chin & 4 & 0 & 0 & 4 \\
& Neck & 1 & 0 & 0 & 1 \\
& Chest & 2 & 2 & 1 & 1 \\
& Abdomen & 2 & 1 & 0 & 3 \\
& Fingers & 3 & 0 & 0 & 3 \\
& Hands & 17 & 0 & 0 & 17 \\
& Forearms & 2 & 0 & 0 & 3 \\
& Lumbosacral & 0 & 1 & 1 & 2 \\
& region & & & & \\
& Forefoot & 3 & 0 & 0 & 3 \\
& Legs & 1 & 1 & 0 & 1 \\
Total & Thigh & 2 & 1 & 0 & 3 \\
\hline
\end{tabular}

TABLE 3: Distribution of peri-graft halo and post-inflammatory hyperpigmentation in 61 treated lesions

\begin{tabular}{ll}
\hline Complication & Frequency (\%) \\
\hline Only Peri-graft halo & $13(21.31)$ \\
Only Post-inflammatory hyper pigmentation & $7(11.48)$ \\
Both & $4(6.56)$ \\
Neither & $37(60.65)$ \\
Total & $\mathbf{6 1 ( 1 0 0 . 0 0 )}$ \\
\hline
\end{tabular}

TABLE 4: Complications of treated lesions based on location

\begin{tabular}{llcc}
\hline Location & Peri-graft halo & $\begin{array}{c}\text { Post-inflammatory } \\
\text { hyperpigmentation }\end{array}$ \\
& Forehead & 4 & 3 \\
& Eyelids & 1 & 0 \\
& Cheek & 0 & 0 \\
& Chin & 1 & 1 \\
& Neck & 0 & 0 \\
& Chest & 1 & 0 \\
& Abdomen & 0 & 1 \\
& Fingers & 0 & 0 \\
& Hands & 7 & 5 \\
& Forearms & 1 & 0 \\
& Lumbosacral & 0 & 0 \\
& Forefoot & 1 & 0 \\
Total & Legs & 0 & 1 \\
& Thigh & 1 & 0 \\
\hline
\end{tabular}

recipient sites. Erythema and PIH occurred on all donor sites, lasting for 3-6 months, but no scar formation was noted.

\section{DISCUSSION}

Results showed that excellent repigmentation occurred in $53(86.9 \%)$ of the grafted sites prepared by motor-driven carbide bur within 6-8 months' followup; good to moderate repigmentation occurred in 6 $(9.8 \%)$, while poor repigmentation occurred in 2 $(3.3 \%)$ cases of grafted skin.
In SBEG, although incomplete removal of recipient epidermis and damaged or necrotic layer under the grafted epidermis are involved in partial or complete graft failure, the most common cause of graft failure is improper immobilization and separation of the grafted epidermis in the first few days after grafting. ${ }^{3}$ In our study, partial graft failures occurred at the lumbosacral and chest recipient sites where graft immobilization was difficult. Although poor repigmentation outcomes have been reported on areas such as eyelids and dorsum of hands through Suction Blistering Epidermal Graft (SBEG), in our study, this method led to excellent repigmentation in all vitiligenous lesions affecting the eyelids and dorsum of hands and fingers.

We consider that excellent repigmentation in the eyelids, dorsum of hands and fingers may be related to accurate preparation of recipient sites and proper immobilization of grafted epidermis, via hand split and complete eye occlusion for 5-7 days.

Postinflamatory hyperpigmentation (PIH) is a common problem during the first few months following epidermal graft procedures., ${ }^{5,6}$ In our study, $\mathrm{PIH}$ occurred in $11(18 \%)$ lesions during the first two months but most faded completely during the sixth month. However, in $2(3.3 \%)$ lesions, partial PIH was observed even after the end of follow-up. In previous studies, transient PIH was observed in $2-62 \%$ of patients, caused by inflammation induced by the preparation method for the recipient site and skin typing of patients. ${ }^{5,6}$ Greater inflammation leads to more $\mathrm{PIH}$ at the grafted epidermis. ${ }^{1}$ The motor-driven carbide bur mechanically removes the epidermis and leaves the dermis intact, while there is less inflammation compared with LN and topical PUVA.

Scar and keloid formation was not observed at the different recipient sites because application of the suggested fraise was associated with minimal manipulation of recipient site. In contrast, scar formation has been reported as a complication of recipient site preparation by LN, motor-driven diamond fraise, and $\mathrm{CO}_{2}$ laser. ${ }^{5,8,9}$

Perigraft halo occurred in (17) $27.9 \%$ of recipient sites, in the form of a 1-2 mm depigmented area. It was observed within the first three months and repaired by pigment spreading during the follow-up period. Perigraft halo usually occurs due to shrinkage of suctioned donor epidermis after removing the sustained negative pressure, insufficient abrasion of recipient epidermis and inhibitory effects of $\mathrm{LN}$ on the melanocytes of peripheral pigmented epidermis. ${ }^{8,9}$ The motor-driven carbide bur precisely abrades the recipient epidermis even in complex-shaped lesions, based on clinical observations such as pinpoint bleeding or histologic analysis of lesions. Furthermore, 
perigraft halo is not due to insufficient abrasion of recipient epidermis (Figures 2 and 3). However, with respect to bullae induced by LN and topical PUVA, precise removal of depigmented epidermis may be difficult and LN may lead to some inhibitory effects on the melanocytes of peripheral pigmented epidermis. In addition, LN may lead to severe edema of some recipient sites including the eyelids and genitalia. ${ }^{5,89}$ Although topical PUVA leads to easy separation of epidermis from the recipient site, it entails complications such as severe erythema, $\mathrm{PIH}$ and prolonged color mismatch of the grafted epidermis. ${ }^{5,6} \mathrm{LN}$ and topical PUVA must be performed two days before transplantation and they are not reliable methods for complete removal of depigmented epidermis, especially in complex-shaped lesions. ${ }^{1,3,6}$ Moreover, since blisters are not usually complete on all suctioned donor sites, it is difficult for dermatologists to match the harvested epidermis with the size of the previously prepared recipient site. Conversely, in SBEG using a motor-driven carbide bur, the recipient site was prepared when bullae formation became complete at the donor sites and harvested epidermis was matched with the recipient site.

Preparing the recipient site by suction pump is time-consuming and it is difficult to apply the suction tips on some recipient sites, including the periorbital and perinasal regions, as well as the fingers and genitalia. ${ }^{5,6}$ In addition, bullae formation caused by the suction pump is not always complete and some islands of depigmented epidermis may remain attached to the underlying dermis. Thus, it is not a reliable method for completely removing the recipient epidermis. PIH is a well-known complication of SBEG at the donor site, which may also occur at the recipient site because of the suction pump. Furthermore, it is an uncomfortable method for the patient who endures suction tips at the donor and recipient sites, as well as for the dermatologist who prepares multiple and complex-shaped recipient sites. ${ }^{5}$ As shown, the motor-driven carbide bur is an easy and reliable method, even for complex-shaped lesions on the hands, fingers and periorbital areas.

Abrasion of the recipient site by a motor-driven diamond fraise is a simple and effective method. Precise abrasion of depigmented epidermis in any anatomic area makes it reliable, though it is a timeconsuming procedure. Abrasion of the epidermis using diamond fraises, which are designed for abrasion of the dermis, requires skilled hands and achieving a uniform depth can be difficult. Furthermore, unnecessary abrasion of the dermis may lead to scar formation, especially on the scar-prone sites. ${ }^{8}$ However, one of the characteristics of the suggested fraise is that it creates a minimally traumatized under- lying dermis, though care must be taken because application of this fraise by unskilled hands, as well as high speed or extra pressure during the abrasion of the epidermis, creates additional heat and raises the temperature within the underlying dermis, although it cannot remove the dermis. ${ }^{10}$

The Erbium-YAG laser and pulsed $\mathrm{Co}_{2}$ laser are reliable methods for precise ablation of depigmented epidermis from any anatomical site., $\mathrm{Oh}$ CK et al. suggested that subsequent thermal necrosis of $\mathrm{CO}_{2}$ laser does not affect satisfactory graft take, while Kahn et al. demonstrated similar histology in skin graft adherence between the dermabrasion and $\mathrm{CO}_{2}$ laser. ${ }^{11,12}$ However, Lee DY noted better graft uptake and faster repigmentation through mechanical removal of depigmented epidermis for preparation of the recipient site, compared with the $\mathrm{CO}_{2}$ laser. ${ }^{13}$ Moreover, in practice, the $\mathrm{CO}_{2}$ laser causes homeostasis in the superficial microvasculature of the papillary dermis and thermal damage may lead to a thin, thermal necrotic layer beneath the grafted epidermis. In addition, the $\mathrm{Co}_{2}$ laser entails complications such as scar formation and pigmentry changes, especially on the dorsum of hands and fingers. Nevertheless, lasers may not be available in some clinics and departments. Hence, using our method could be an appropriate alternative for preparing recipient sites, even in scarprone areas such as the trunk and limbs.

Ultrasonic surgical aspirators that remove only the epidermis of the recipient site have been reported to be very successful, with excellent repigmentation at all grafting sites, and can be an alternative to motordriven carbide burs. ${ }^{8}$ However, the motor-driven dermabrader is a standard device in dermatology and it seems cost-effective to substitute a diamond fraise with a dental lab carbide bur.

\section{CONCLUSION}

SBEG is a multi-stage procedure and the preparation method for the recipient site plays an important role in the final repigmmentation rate, cosmetic outcome and associated complications. Our results demonstrated that grafting epidermis on erosions made by motor- driven carbide bur is an easy and reliable method for refractory and stable vitiligo; repigmentation was excellent, $\mathrm{PIH}$ and perigraft halo were mild and transient.

We used this new equipment for high-risk areas such as the trunk and limbs, without any risk of keloids or hypertrophic scars. $\square$

\section{ACKNOWLEDGMENTS}

The authors wish to thank Dr Hamid Madani, associated professor at the department of pathology; Kermanshah University of Medical Sciences. 


\section{REFERENCES}

1. Falabella R. Surgical approaches for stable vitiligo. Dermatol Surg. 2005;31:1277-84.

2. Radmanesh M, Ebrahimi A. Double syringe blistering by adding a three-way connector for grafting stable vitiligo patches. J Dermatol Treat. 2000;11:43-6.

3. de Leeuw J, Assen YJ, van der Beek N, Bjerring P, Martino Neumann HA. Treatment of vitiligo with khellin liposomes, ultraviolet light and blister roof transplantation. J Eur Acad Dermatol Venereol. 2011;25:74-81.

4. Rusfianti M, Wirohadidjodjo YW.Dermatosurgical techniques for repigmentation of vitiligo. Int J Dermatol. 2006;45:411-7.

5. van Geel N, Ongenae K, Naeyaert JM. Surgical techniques for vitiligo: a review. Dermatology. 2001;202:162-6.

6. Njoo MD, Westerhof W, Bos JD, Bossuyt PM. A systematic review of autologous transplantation methods in vitiligo. Arch Dermatol. 1998;134:1543-9.

7. Khunger N, Kathuria SD, Ramesh V. Tissue grafts in vitiligo surgery - past, present, and future. Indian J Dermatol. 2009;54:150-8.

8. Tsukamoto K, Osada A, Kitamura R, Ohkouchi M, Shimada S, Takayama O. Approaches to repigmentation of vitiligo skin: new treatment with ultrasonic abrasion, seed-grafting and psoralen plus ultraviolet A therapy. Pigment Cell Res. 2002;15:331-4.

9. Kim HY, Kang KY. Epidermal grafts for treatment of stable and progressive vitiligo. J Am Acad Dermatol. 1999;40:412-7.

10. Issing K, Friederich HC. Telethermographic aspects of dermabrasion including the management of postoperartive wounds. Z Hautkr. 1987;62:1007-28.

11. Oh CK, Cha JH, Lim JY, Jo JH, Kim SJ, Jang HS, et al. Treatment of vitiligo with suction epidermal grafting by the use of an ultrapulse $\mathrm{CO} 2$ laser with a computerized pattern generator. Dermatol Surg. 2001;27:565-8.

12. Kahn AM, Ostad A, Moy RL. Grafting following short-pulse carbon dioxide laser de-epithelialization. Dermatol Surg. 1996;22:965-7.

13. Lee DY, Park JH, Choi SC, Lee JH. Comparison of recipient site preparations in epidermal grafting for vitiligo: suction blister and CO2 laser. J Eur Acad Dermatol Venereol. 2009;23:1448-9.
MAILING ADDRESS:

Hossein Kavoussi

Hajdaiee dermatology clinic

Golestan Blvd

Kermanshah, Iran.

E-mail:hkawosi@kums.ac.ir

How to cite this article: Ebrahimi A, Radmanesh M, Kavoussi H. Recipient site preparation for epidermal graft in stable vitiligo by a special fraise. An Bras Dermatol. 2015;90(1):55-60. 